\title{
Histone deacetylase inhibitor valproic acid attenuates high glucose-induced endoplasmic reticulum stress and apoptosis in NRK-52E cells
}

\author{
XINYI SUN ${ }^{1}$, YUMAN SUN ${ }^{1}$, SITONG LIN $^{1}$, YAN XU $^{1}$ and DONGMING ZHAO ${ }^{2}$ \\ ${ }^{1}$ Endocrine Department and ${ }^{2}$ Cardiovascular Department, Affiliated Hospital \\ of Beihua University, Chuanying, Jilin 132011, P.R. China
}

Received March 5, 2019; Accepted April 15, 2020

DOI: $10.3892 / \mathrm{mmr} .2020 .11496$

\begin{abstract}
Previous studies have demonstrated that valproic acid (VPA), a histone deacetylase inhibitor, alleviates diabetic nephropathy (DN). However, the biological mechanisms underlying this protective effect remains unclear. This study aimed to investigate the effects of histone deacetylase inhibitor VPA on hyperglycemic induction of NRK-52E cell ERS and apoptosis. Endoplasmic reticulum stress (ERS)-related apoptosis is involved in DN, and improving ERS may delay the symptoms of DN. Histone deacetylase regulates gene transcription or expression of ERS-related proteins. The present study established an ERS model by treating the rat renal tubular epithelial cells NRK-52E with high glucose (HG) and investigated the effects of VPA on the apoptosis of the NRK-52E cells. HG stimulation significantly increased the protein levels of the ERS-related proteins including glucose regulated protein 78 (GRP78), activating transcription factor 4 (ATF4), C/EBP homologous protein (CHOP), caspase-12 and phosphorylated (p)-JNK. VPA treatment further upregulated GRP78 expression and attenuated the levels of ATF4, CHOP, caspase-12 and p-JNK. Notably, HG markedly promoted apoptosis of NRK-52E cells by regulating the protein levels of Bax, cleaved caspase- 3 and Bcl-2, which was attenuated by simultaneous VPA treatment. Mechanistically, VPA increased the total acetylation levels of histone H4 in NRK-52E cells and increased the histone H4 acetylation of the GRP78 promoter region. In conclusion, VPA attenuated HG-induced ERS and apoptosis in NRK-52E cells, which may be due to the regulation of acetylation levels of ERS-related proteins. In addition, the present study suggested that HDACIs are promising drugs for treating patients with DN.
\end{abstract}

Correspondence to: Dr Dongming Zhao, Cardiovascular Department, Affiliated Hospital of Beihua University, 12 Jiefang Middle Road, Chuanying, Jilin 132011, P.R. China

E-mail: zdmjls@126.com

Key words: histone deacetylase inhibitor, valproic acid, diabetic nephropathy, NRK-52E cells, endoplasmic reticulum stress, apoptosis

\section{Introduction}

Diabetes mellitus is one of the most common chronic diseases in nearly all countries $(1,2)$, in 2010 , which collectively killed globally an estimated 12.9 million people (3). According to the World Health Organization Global Report, approximately 1.6 million people worldwide died due to diabetes in 2016, and it is estimated that 425 million people are living with diabetes worldwide, this number is predicted to rise to approximately 629 million globally in 2045 (4). Diabetic nephropathy (DN) is one of the most significant complications of diabetes and the main cause of end-stage renal failure (5), which seriously threatens the health of patients and brings a heavy economic burden to the families and society. Therefore, the treatment of $\mathrm{DN}$ has become a topic of concern.

The exact etiology of DN is unknown; however, various postulated mechanisms, including renal hemodynamic changes, abnormal glucose metabolism, genetic factors, cytokines, oxidative stress and other factors $(6,7)$, have been proposed. Recent research on the causes of DN has demonstrated that DN is closely associated with apoptosis (8). The apoptosis of diabetic kidney tissue cells is related podocytes and mesangial cells, especially renal tubular epithelial cells are significantly associated with apoptosis (9-11). The endoplasmic reticulum stress (ERS) pathway is one of the apoptotic pathways. Studies have demonstrated that DN initiates ERS-related ER-associated death (ERAD) and induces apoptosis (12-14). For example, the role of reactive oxygen species-mediated ERS has been revealed in contrast-induced renal tubular cell apoptosis (15). During the diabetic process, ERS is induced by factors such as high sugar, excess nutrients, increased free fatty acids and oxidative stress. These persistent and unmotivated stimuli eventually cause irreversible ERS, initiate ERAD, and amplify the cascade effects of apoptosis in renal tubular epithelial cells (13). Qi et al have reported that the regulation of the ERS inflammatory response attenuates DN in diabetic rats (16). Therefore, effective intervention against ERS may benefit the treatment of DN.

Chromatin structural changes affect the regulation of gene expression, and the acetylation of histones regulates gene transcription (17). It has been demonstrated that histone acetylation regulates the transcription of ERS-related 
genes $(18,19)$. Valproic acid (VPA) is a representative drug of the non-selective histone deacetylase inhibitors (HDACIs) and is clinically used as anticonvulsant and sedative (16). Studies have demonstrated that VPA reduces the expression of transforming growth factor (TGF) $\beta$ in DN, thereby reducing renal fibrosis (20-23). Studies have also demonstrated that VPA reduces mesenchymal transition of NRK-52E cells $(24,25)$. Therefore, VPA mitigates DN; however, to the best of our knowledge, the specific mechanisms are not fully elucidated.

Our previous study demonstrated significant apoptosis of renal cell tissue and changes of ERS-related proteins in renal tubular epithelial cells (26). A high concentration of glucose is an ERS-inducing factor that mimics the natural, pathological process of clinical DN. Therefore, in the present study, the renal tubular epithelial cells NRK-52E were selected as the model cell line to evaluate the impacts of high concentration of glucose on ERS and apoptosis. To determine whether and how the effect of VPA on DN is related to ERS-related apoptosis, the present study investigated the regulatory mechanisms of VPA on histone acetylation.

\section{Materials and methods}

Cell culture. The rat renal tubular epithelial cell line NRK-52E (cat. no. ATCC ${ }^{\circledR}$ CRL-1571 ${ }^{\mathrm{TM}}$ ) was purchased from the American Type Culture Collection. The cells were cultured with either high-glucose (HG; $25 \mathrm{mM}$ glucose) DMEM (Gibco; Thermo Fisher Scientific, Inc.) or low-glucose (5 mM glucose) DMEM. Culture medium, containing $10 \%$ fetal bovine serum (Gibco; Thermo Fisher Scientific, Inc.), was supplemented with $100 \mathrm{U} / \mathrm{ml}$ penicillin (Gibco; Thermo Fisher Scientific, Inc.) and $100 \mu \mathrm{g} / \mathrm{ml}$ streptomycin (Gibco; Thermo Fisher Scientific, Inc.). The culture flasks (Beyotime Institute of Biotechnology) were placed in a humidified incubator (Sanyo Electric Co., Ltd.) at $37^{\circ} \mathrm{C}$ with $5 \% \mathrm{CO}_{2}$. The culture medium was changed every 1-2 days, and the cells were passaged every 2-3 days according to cell confluence.

Titration of VPA and cell treatment. NRK-52E cells were seeded in a 96-well plate (cat. no. JY92-2D Beyotime Institute of Biotechnology) at a density of $1 \times 10^{4}$ cells $/ 100 \mu \mathrm{l} /$ well . The cells were cultured for $24 \mathrm{~h}$ to achieve a logarithmic growth phase and different concentrations of VPA $(0.1,1,10$ and $100 \mathrm{nM}$ ) were administered. Three replicate wells were set for each concentration, and three negative control wells and blank control wells were also included. After culturing for $48 \mathrm{~h}, 10 \mu \mathrm{l}$ MTT saline solution was added to each well, and the culture was continued for $4 \mathrm{~h}$. The absorbance (A) value of each well was measured at a wavelength of $450 \mathrm{~nm}$ by a microplate reader (Tecan Group Ltd.) to calculate the cell growth inhibition rate. Cell growth viability rate $(\%)$ at different concentrations $=(A$ value of VPA group $-A$ value of blank group)/(A value of negative control group-A value of blank group) $\mathrm{x} 100 \%$. The selected concentration of the drug was $10 \mathrm{nM}$ (Fig. 1). The cells in the logarithmic growth phase were divided into four groups: i) Control: Cells were cultured with low glucose (5 mM) DMEM; ii) Control+VPA: Cells were cultured with low glucose (5 mM) DMEM supplemented with 10 nM VPA (Sigma-Aldrich: Merck KGaA); iii) HG: Cells were cultured with HG (25 mM) DMEM; and iv) HG+VPA:
Cells were cultured with HG (25 mM) DMEM supplemented with $10 \mathrm{nM}$ VPA.

Total cellular protein extraction. Harvested cells were washed twice with pre-cooled PBS, and transferred to a 1.5-ml EP tube. After centrifugation at $1,000 \mathrm{~g} / 5 \mathrm{~min}$ at $4^{\circ} \mathrm{C}$, the supernatant was discarded and $150 \mu \mathrm{l}$ RIPA protein lysis buffer (50 mM Tris- $\mathrm{HCl}, \mathrm{pH} 7.5,150 \mathrm{mM} \mathrm{NaCl}, 1 \mathrm{mM}$ $\mathrm{Na}_{2}$ EDTA, $1 \mathrm{mM}$ EDTA, $1 \%$ Triton, $2.5 \mathrm{mM}$ sodium pyrophosphate, $1 \mathrm{mM} \beta$-glycerophosphate, $1 \mathrm{mM} \mathrm{Na} \mathrm{VO}_{4}, 1 \mathrm{mM}$ $\mathrm{NaF}, 1 \mu \mathrm{g} / \mathrm{ml}$ leupeptin and $1 \mathrm{mM}$ PMSF; Beyotime Institute of Biotechnology) supplemented with cocktails of protease inhibitor and phosphatase inhibitor (Amresco) were added to each EP tube. All the operations were performed on ice to avoid protein degradation. The cells were subjected to ultrasonication with an ultrasonic cell crusher (Ningbo Xinzhi Technology Co., Ltd.) three times for $5 \mathrm{sec}$ and then the cells were allowed to stand at $4^{\circ} \mathrm{C}$ for $45 \mathrm{~min}$. Next, the cells were centrifuged at $1,000 \mathrm{x} \mathrm{g}$ for $15 \mathrm{~min}$ at $4^{\circ} \mathrm{C}$, and the supernatant was taken and dispensed in EP tubes. After the protein concentration was measured with a Pierce BCA kit (Thermo Fisher Scientific Inc.), every $100 \mu 1$ protein sample was mixed with $25 \mu \mathrm{l}$ of $5 \mathrm{x}$ loading buffer. The mixture was boiled in water at $95^{\circ} \mathrm{C}$ for $10 \mathrm{~min}$ and then incubated at $37^{\circ} \mathrm{C}$ for $10 \mathrm{~min}$ in a water bath (Shanghai Bo Xun Industrial Co., Ltd.). The samples that were not immediately used were stored at $-20^{\circ} \mathrm{C}$.

Western blot analysis. Protein lysates (30-50 $\mu \mathrm{g}$ ) were resolved by $12 \%$ SDS polyacrylamide gel electrophoresis (Beyotime Institute of Biotechnology) and transferred onto immobilon-P transfer membranes (EMD Millipore). The membranes were blocked with $5 \%$ non-fat milk powder in buffer [10 mM Tris-HCl, $(\mathrm{pH} 7.6), 100 \mathrm{mM} \mathrm{NaCl}$ and $0.1 \%$ Tween 20] for $2 \mathrm{~h}$ at room temperature and then incubated with the desired primary antibody, including polyclonal rabbit anti-human glycine-rich RNA-binding protein 78 (GRP78; cat. no. ab21685, 1:1,000), polyclonal rabbit anti-human caspase-12 (cat. no. ab4051, 1:500), rabbit polyclonal anti-caspase-3 (cat. no. ab13847, 1:500), mouse anti-human monoclonal C/EBP homologous protein (CHOP; cat. no. ab11419, 1:500), monoclonal rat anti-human B-cell lymphoma 2 (Bcl-2; cat. no. ab182858, 1:2,000), rabbit monoclonal anti-Bcl-2-associated X protein (Bax; cat. no. ab32503, 1:3,000), polyclonal rabbit anti-phosphorylated JNK (p-JNK; cat. no. ab4821, 1:1,000), monoclonal mouse anti-activating transcription factor 4 (ATF4; cat. no. ab23760, 1:1,000) or monoclonal mouse anti- $\beta$-actin $(1: 1,000$; cat. no. ab6276; all antibodies were obtained from Abcam) overnight at $4^{\circ} \mathrm{C}$, followed by incubation with a horseradish peroxidase (HRP)-conjugated secondary antibody (Hangzhou HuaAn Biotechnology Co., Ltd.) at a 1:2,000 dilution for $90 \mathrm{~min}$ at room temperature. The immunoreactive bands were visualized with 3,3'-diaminobenzidine (Sigma-Aldrich; Merck KGaA). The representative bands were measured by a Tanon GIS gel imager system (Tanon Science \& Technology Co., Ltd.) and analyzed. The levels of proteins were normalized to those of $\beta$-actin, and the ratios are presented as the mean \pm standard deviation of three independent experiments. Protein levels were quantified by densitometry using the Quantity One 1-D software (version 4.4.02; Bio-Rad Laboratories, Inc.). 
Total histone $\mathrm{H} 4$ acetylation assay. The fast detection of total histone $\mathrm{H} 4$ acetylation in NRK-52E cells was performed using the EpiQuik ${ }^{\mathrm{TM}}$ Total Histone H4 Acetylation Detection Fast kit (Colorimetric) (cat. no. P-4032; Epigentek Group Inc.), as previously described.

Chromatin immunoprecipitation assay (CHIP). CHIP assays were performed using a CHIP assay kit (EMD Millipore), according to the manufacturer's instructions. Briefly, NRK-52E cells were treated as indicated, cross-linked with formaldehyde and sonicated. Resulting cell lysates (input) were immunoprecipitated with the anti-acetylated-histone $\mathrm{H} 4$ antibody (cat. no. ab109463; Abcam). The precipitated protein-DNA complexes (IP) were subjected to proteinase treatment. The following primers were used: GRP78 forward, 5'-CTCGAG GAAGGGCATAAGAGCATCA-3' and reverse, 5'-CCGCTT CTCCTCAGGTTCCGGCTGT-3'; CHOP forward, 5'-ACT GACAACGACAAGACCCC-3' and reverse, 5'-AGTCACAGC CAGTATCGAGC-3'; and GAPDH forward, 5'-ACAACCTGG TCCTCAGTGTAGCC-3' and reverse, 5'-AAGGTCATCCCA GAGCTGAACGG-3'.

Statistical analysis. Data are expressed as the mean \pm standard error of the mean (SEM) of three independent experiments, each conducted in triplicate. One-way analysis of variance (ANOVA) followed by a Dunnett's multiple comparison test was performed to compare differences between multiple groups. All comparisons were performed with GraphPad Prism (version 5, GraphPad Software Inc.). $\mathrm{P}<0.05$ was considered to indicate a statistically significant difference.

\section{Results}

VPA attenuates HG-induced ERS in NRK-52E cells. First, a titration of VPA concentrations by MTT assays was performed to select the optimal concentration of VPA for treating NRK-52E cells. Following 48 h of culture, the cells treated with $100 \mathrm{nM}$ VPA had a significantly decreased viability compared with the control cells under normal glucose conditions (Fig. 1A; $\mathrm{P}<0.05)$. The viability of cells cultured with the HG medium significantly increased slightly with the addition of $10 \mathrm{nM}$ VPA $(\mathrm{P}<0.05)$, but significantly decreased with the addition of $100 \mathrm{nM}$ VPA $(\mathrm{P}<0.05$; Fig. 1B). Therefore, the optimal working concentration of VPA was determined to be $10 \mathrm{nM}$.

To investigate the effects of VPA on ERS induced by HG in NRK-52E cells, the expression levels of the ERS-related proteins GRP78, ATF4, CHOP and caspase-12 in NRK-52E cells cultured under $\mathrm{HG}$ conditions and treated with $10 \mathrm{nM}$ VPA were determined. Compared with the control group, the expression of the GRP78 protein in the HG group was significantly increased $(\mathrm{P}<0.05)$, while supplementation of VPA further increased the protein levels of GRP78 (P<0.05; Fig. 2A). The expression of ATF4 was also significantly increased after stimulation with HG for $48 \mathrm{~h}$, while supplementation of VPA (the HG+VPA group) significantly reversed this effect $(\mathrm{P}<0.05$; Fig. 2A). A similar trend of CHOP or caspase-12 expression was obtained (Fig. 2A), and similar to ATF4, VPA was demonstrated to significantly reduce the expression levels of $\mathrm{CHOP}$ and caspase-12 $(\mathrm{P}<0.05)$. By contrast, VPA at $10 \mathrm{nM}$ did not affect the expression of these molecules under the low glucose


Figure 1. Optimizing the concentration of VPA for treating NRK-52E cells. NRK-52E cells seeded in a 96-well plate were cultured for $24 \mathrm{~h}$ to achieve a logarithmic growth phase, and different concentrations of VPA $(0.1,1,10$ and $100 \mathrm{nM}$ ) were added to the culture medium. After culturing for $48 \mathrm{~h}$, the cells were subjected to MTT assays for determining cell viability. (A) Low $(5 \mathrm{mM})$ glucose medium or $(\mathrm{B})$ high $(25 \mathrm{mM})$ glucose medium was used for cell culture. ${ }^{*} \mathrm{P}<0.05$

condition. These results suggested that VPA attenuated the HG-induced ERS in NRK-52E cells.

VPA attenuates apoptosis of NRK-52E cells cultured under the $H G$ condition. Compared with the control group, HG stimulation also significantly enhanced the ratio of $\mathrm{p}-\mathrm{JNK} / \mathrm{JNK}$ in NRK-52E cells, and VPA treatment significantly attenuated the increase of p-JNK/JNK upon HG culture (Fig. 2A). Since $\mathrm{JNK}$ activation is reported to be involved in ER stress-induced cell death, and since both caspase-12 and CHOP are key ERS-related apoptotic proteins, the present study hypothesized that VPA may affect ERS-induced apoptosis. To observe the effect of VPA on the apoptosis of NRK-52E cells induced by $\mathrm{HG}$, western blotting was performed to detect the expression levels of apoptosis-related proteins, such as Bax, cleaved-caspase-3 and Bcl-2 (Fig. 2B). Compared with the control group, HG stimulation in NRK-52E cells significantly enhanced the expression of the apoptosis-promoting factors Bax and cleaved caspase-3, but reduced the expression of the apoptosis-inhibiting factor Bcl-2 (Fig. 2B). In addition, VPA exhibited no effects on the expression of these factors in cells cultured under low glucose condition, whereas VPA treatment in cells under HG condition significantly attenuated the effects of HG-induced changes in protein levels of these molecules 

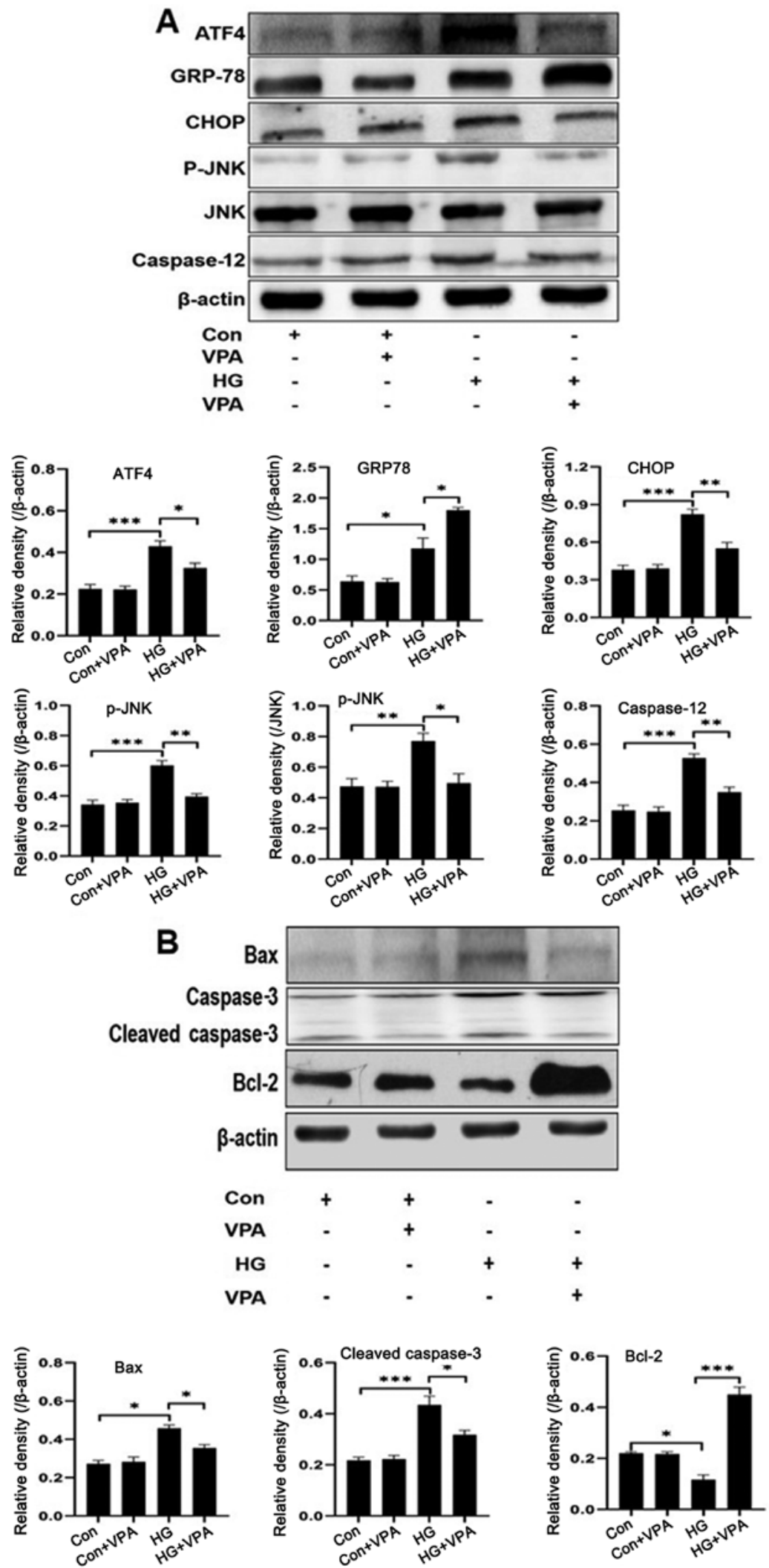

Figure 2. Western blotting revealed that VPA attenuates ER stress-induced apoptosis in NRK-52E cell upon high glucose treatment. NRK-52E cells were treated as indicated for $48 \mathrm{~h}$, and cell lysates were collected to determine the protein levels of (A) ER stress-related proteins (GRP78, ATF4, CHOP, caspase-12 and p-JNK) and (B) apoptosis-related molecules (Bax, cleaved caspase-3 and Bcl-2) by western blot analyses. ${ }^{*} \mathrm{P}<0.05,{ }^{* * *} \mathrm{P}<0.01,{ }^{* * *} \mathrm{P}<0.001$. VPA, valproic acid; GPR78, ATF4, activating transcription factor 4; CHOP, C/EBP homologous protein; HG, high glucose; p-, phosphorylated.

(Fig. 2B). Therefore, these results demonstrated that VPA is an antagonistic factor against HG-induced apoptosis in NRK-52E cells.
Investigating the role of VPA in histone acetylation on $H G$-induced ERS and apoptosis in NRK-52E cells. VPA inhibits histone deacetylase and promotes histone acetylation, which is 


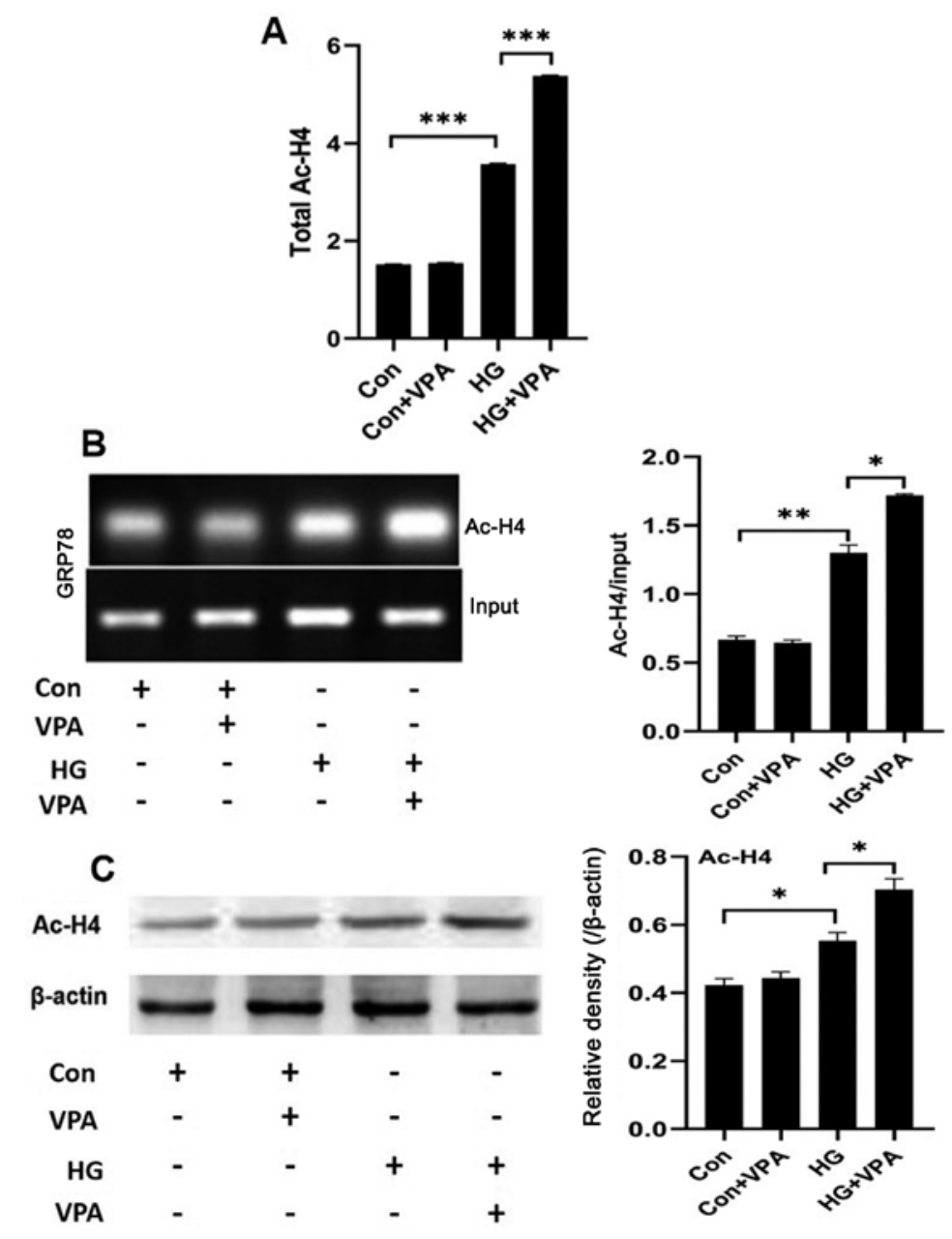

Figure 3. VPA enhances the acetylation levels of histone H4 in HG-induced NRK-52E cells. (A) Total histone H4 acetylation in NRK-52E cells was detected using an EpiQuik ${ }^{\mathrm{TM}}$ Total Histone H4 Acetylation Detection Fast kit. $\mathrm{n}=3$ for each group. (B) The effect of $10 \mathrm{nM}$ VPA on histone H4 acetylation of the GRP78 gene promoter was determined by the CHIP assay in the HG $(25 \mathrm{mmol} / \mathrm{l})$ and low glucose $(5 \mathrm{mmol} / \mathrm{l})$ groups. The upper images represent PCR results from one of at least three independent experiments with similar results, while the lower bar graphs are summarized results by quantitative densitometry analyses. (C) Total histone H4 acetylation in NRK-52E cells was detected by western blotting. " $\mathrm{P}<0.05,{ }^{* *} \mathrm{P}<0.01,{ }^{* * * *} \mathrm{P}<0.001$. VPA, valproic acid; HG, high glucose; Ac- $\mathrm{H} 4$, histone $\mathrm{H} 4$ acetylation.

involved in the transcriptional activity of genes (27). Thus, the role of VPA was evaluated by investigating whether and how it affected histone acetylation in NRK-52E cells under the HG $(25 \mathrm{mmol} / \mathrm{l})$ and low-glucose $(5 \mathrm{mmol} / \mathrm{l})$ culture conditions. First, a colorimetric assay was used to determine the total acetylation levels of histone H4 in NRK-52E cells. Compared with the control group, the total histone $\mathrm{H} 4$ acetylation level in the HG group was significantly higher, while the treatment of $\mathrm{HG}$ in combination with $10 \mathrm{nM}$ VPA (the HG+VPA group) further increased the total histone $\mathrm{H} 4$ acetylation levels (Fig. 3A and C). Next, CHIP assays were performed to measure the histone $\mathrm{H} 4$ acetylation levels in the promoter region of the ERS key protein GRP78. The acetylation level of histone $\mathrm{H} 4$ in the GRP78 promoter region was significantly increased in the HG group compared with that of the control group and was further increased in the HG+VPA group (Fig. 3B). Taken together, VPA exhibits a role of broad enhancement on histone $\mathrm{H} 4$ acetylation in NRK-52E cells.

\section{Discussion}

As a major chronic complication of diabetes, DN gains more and more attention as the number of $\mathrm{DN}$ cases increases as the incidence of diabetes increases. A total of $30-40 \%$ of patients with diabetes eventually develop DN (28); therefore, how to effectively treat DN has become a focus of concern. Studies have highlighted the crucial roles of ERS and apoptosis in the pathogenesis of DN (12-14). In the present study, this notion is further strengthened by the findings that HG-stimulated NRK-52E renal tubular epithelial cells underwent ERS and that the HDACI VAP attenuated the effects of HF, at least partially through the regulation on the acetylation of histone $\mathrm{H} 4$.

Various studies have demonstrated that DN is closely related to the apoptosis-associated ERS pathway, and ERS has become one of the pathogenic processes of DN (29,30). Diabetes hyperglycemia stimulates ERS in renal tubular epithelial cells or podocytes, and causes apoptosis $(9,31,32)$. Our previous study demonstrated increased expression of the ERS-related protein GRP78 and increased expression of ERS-related apoptosis proteins CHOP and caspase- 12 in DN rat kidneys compared to wild type rats (26). Renal apoptotic cells were mainly found in renal tubular epithelial cells (33). Knockout of the ERS-related protein GRP78 in mice leads to phenotypes that include renal tubular atrophy, interstitial fibrosis and glomerular sclerosis (34). Our previous study also demonstrated that ERS and apoptosis of 
DN rats mainly occurred in the renal tubules (26); accordingly, the present study investigated the effects of HG on ERS of the NRK-52E renal tubular epithelial cells. The results of the present study demonstrated that compared with the control group the expression of the ERS-related protein GRP78 was increased, and the expression of the ERS-related apoptosis proteins CHOP and caspase-12 was also increased when NRK-52E cells were cultured under HG. This suggested that HG activates the ERS-related apoptosis pathways in NRK-52E cells. Consistent with other relevant research findings, the results of the present study suggested that ERS-induced apoptosis may be one of the causes of kidney failure. Thus, we hypothesize that reducing ERS-related apoptosis may attenuate the development of DN.

The regulation of expression of ERS-related apoptotic proteins is related to chromatin structure, in addition to the regulation of genomic DNA levels (35). Since the acetylation of histones is related to chromatin remodeling, it is also involved in the regulation of gene expression. It has been demonstrated that histone acetylation may be involved in the regulation of ERS-related protein expression (36), and HDACIs are closely associated with cell proliferation, migration and apoptosis (37). VPA is a non-selective HDACI; a recent study demonstrated that it reduces proteinuria in a rat model of renal ischemia-reperfusion injury, thereby alleviating renal damage, and its mechanism may be related to the regulation of TGF- $\beta$ (38). VPA also alleviates DN renal fibrosis (39). The results of the present study demonstrated that compared with those of the control group, the expression levels of the ERS-related protein GRP78, which is an anti-apoptotic protein, was further increased after HG-induced NRK-52E cells were administered VPA, whereas the expression levels of ERS-related pro-apoptotic proteins ATF4, CHOP, caspase-12 and p-JNK were reduced. In addition, VPA also reduced the expression of other pro-apoptotic related proteins such as Bax and cleaved-caspase-3, and increased the expression of the anti-apoptotic protein $\mathrm{Bcl}-2$. In corroboration with the results of the present study, in a model of myocardial ischemia-reperfusion injury, trichostatin A (another type of HDACI) was demonstrated to increase the expression of GRP78 and reduce the expression of the pro-apoptotic proteins CHOP and caspase-12 $(40,41)$. Thus, VPA may impede the development of DN by attenuating ERS-related apoptosis.

The present study also investigated the possible mechanisms of the regulation of ERS-related protein expression by VPA. Zhang et al (42) demonstrated that VPA increases the expression of the ERS-related protein GRP78 by increasing the acetylation level of histone $\mathrm{H} 3$, and reduce the expression of $\mathrm{CHOP}$ and caspase-12, thereby reducing ischemia-reperfusion-associated retinal injury. By contrast, the results of the present study demonstrated that VPA further increased the total acetylation level of histone H4. This may be due to the existence of relatively more acetylation sites of histone $\mathrm{H} 4$ than that of histone $\mathrm{H} 3$ (43). In addition, it has been revealed that HDACIs regulate the promoter of GRP78 in cancer cells and promote the transcription of GRP78 (44). Baumeister et al (45) demonstrated that histone acetyltransferase $\mathrm{p} 300$ binds to the GRP78 promoter to promote GRP78 transcription, and Bown et al (46) also demonstrated that VPA regulates the transcription of ERS-related proteins. These studies suggest that histone acetylation may be involved in the transcriptional regulation of GRP78. Accordingly, the CHIP method was performed to detect the specific $\mathrm{H} 4$ acetylation on the GRP78 gene, and the results demonstrated that the histone H4 acetylation levels of the GRP78 gene promoter were increased. This suggested that VPA may regulate the transcription of the ERS-related gene GRP78 and increases the ability of cells to resist apoptosis. Unfortunately, the present study did not detect altered levels of histone $\mathrm{H} 4$ acetylation at the promoters of CHOP and caspase-12 genes despite repeated attempts. This may be due to the inability of histone acetylation to regulate the transcription of all genes. Although HDACIs work in various tissues, they only regulate the transcription of $\sim 20 \%$ of genes (47-49). HDACIs may be selective for the regulation of particular genes, and the underlying mechanisms require a large and complex panel to be explored in future research.

In summary, the results of the present study revealed that the HDACI VPA attenuated HG-induced ERS and ERS-related apoptosis in NRK-52E cells, which is at least partially associated with VPA-regulated histone $\mathrm{H} 4$ acetylation; thus HDACIs are promising therapeutics for mitigating DN.

\section{Acknowledgements}

Not applicable.

\section{Funding}

This work was supported, in part, by The Health Science and Technology Foundation of Jilin Provincial Health Commission (grant no. 2018J080 to XS), Jin Province Natural Science Education Foundation (grant no. JJKH20200055kJ to XS) and Natural Science Foundation of Jilin Province (grant no. 20200201354JC to XS).

\section{Availability of data and materials}

The datasets used and/or analysed during the current study are available from the corresponding author on reasonable request.

\section{Authors' contributions}

XS conceived and coordinated the study, designed, performed and analyzed the experiments, and wrote the paper. YS, SL, YX and DZ performed the data collection, data analysis and revised the paper. All authors read and approved the final manuscript.

\section{Ethics approval and consent to participate}

Not applicable.

\section{Patient consent for publication}

Not applicable.

\section{Competing interests}

The authors declare that they have no competing interests.

\section{References}

1. Forouhi NG and Wareham NJ: Epidemiology of diabetes. Medicine (Abingdon) 42: 698-702, 2014. 
2. Shaw JE, Sicree RA and Zimmet PZ: Global estimates of the prevalence of diabetes for 2010 and 2030. Diabetes Res Clin Pract 87: 4-14, 2010.

3. Xu Y, Wang L, He J, Bi Y, Li M, Wang T, Wang L, Jiang Y, Dai M, Lu J, et al: Prevalence and control of diabetes in Chinese adults. JAMA 310: 948-959, 2013

4. WHO: Global Report on Diabetes. In: World Health Organization, 2016.

5. Tesch GH: Diabetic nephropathy-is this an immune disorder? Clin Sci (Lond) 131: 2183-2199, 2017

6. Cao Z and Cooper ME: Pathogenesis of diabetic nephropathy. J Diabetes Investig 2: 243-247, 2011.

7. Zhao B, Li H, Liu J, Han P, Zhang C, Bai H, Yuan X, Wang X, Li L, Ma H, et al: MicroRNA-23b targets Ras GTPase-activating protein $\mathrm{SH} 3$ domain-binding protein 2 to alleviate fibrosis and albuminuria in diabetic nephropathy. J Am Soc Nephrol 27: 2597-2608, 2016

8. Anil Kumar P, Welsh GI, Saleem MA and Menon RK: Molecular and cellular events mediating glomerular podocyte dysfunction and depletion in diabetes mellitus. Front Endocrinol (Lausanne) 5: 151, 2014.

9. Habib SL: Diabetes and renal tubular cell apoptosis. World J Diabetes 4: 27-30, 2013.

10. Guo C, Li Y, Zhang R, Zhang Y, Zhao J, Yao J, Sun J, Dong J and Liao L: Protective effect of salidroside against diabetic kidney disease through inhibiting BIM-mediated apoptosis of proximal renal tubular cells in rats. Front Pharmacol 9: 1433, 2018.

11. Zhao X, Liu G, Shen H, Gao B, Li X, Fu J, Zhou J and Ji Q: Liraglutide inhibits autophagy and apoptosis induced by high glucose through GLP-1R in renal tubular epithelial cells. Int J Mol Med 35: 684-692, 2015.

12. Fan Y, Lee K, Wang N and He JC: The role of endoplasmic reticulum stress in diabetic nephropathy.Curr Diab Rep 17: 17, 2017.

13. Cunard R and Sharma K: The endoplasmic reticulum stress response and diabetic kidney disease. Am J Physiol Renal Physiol 300: F1054-F1061, 2011.

14. Cameron NE: Role of endoplasmic reticulum stress in diabetic neuropathy. Diabetes 62: 696-697, 2013.

15. Yang Y, Yang D, Yang D, Jia R and Ding G: Role of reactive oxygen species-mediated endoplasmic reticulum stress in contrast-induced renal tubular cell apoptosis. Nephron Exp Nephrol 128: 30-36, 2014

16. Qi W, Mu J, Luo ZF, Zeng W, Guo YH, Pang Q, Ye ZL, Liu L, Yuan FH and Feng B: Attenuation of diabetic nephropathy in diabetes rats induced by streptozotocin by regulating the endoplasmic reticulum stress inflammatory response. Metabolism 60 594-603, 2011.

17. Verdone L, Agricola E, Caserta M and Di Mauro E: Histone acetylation in gene regulation. Brief Funct Genomic Proteomic 5: 209-221, 2006

18. Chen Y, Tsai YH and Tseng SH: HDAC inhibitors and RECK modulate endoplasmic reticulum stress in tumor cells. Int J Mol Sci 18: 258, 2017.

19. Donati G, Imbriano C and Mantovani R: Dynamic recruitment of transcription factors and epigenetic changes on the ER stress response gene promoters. Nucleic Acids Res 34: 3116-3127, 2006.

20. Van Beneden K, Geers C, Pauwels M, Mannaerts I, Wissing KM, Van den Branden $\mathrm{C}$ and van Grunsven LA: Comparison of trichostatin $\mathrm{A}$ and valproic acid treatment regimens in a mouse model of kidney fibrosis. Toxicol Appl Pharmacol 271: 276-284, 2013.

21. Khan S, Jena G and Tikoo K: Sodium valproate ameliorates diabetes-induced fibrosis and renal damage by the inhibition of histone deacetylases in diabetic rat. Exp Mol Pathol 98: 230-239, 2015.

22. Kochar DK, Rawat N, Agrawal RP, Vyas A, Beniwal R, Kochar SK and Garg P: Sodium valproate for painful diabetic neuropathy: A randomized double-blind placebo-controlled study. QJM 97: 33-38, 2004.

23. Khan S, Bhat ZR and Jena G: Role of autophagy and histone deacetylases in diabetic nephropathy: Current status and future perspectives. Genes Dis 3: 211-219, 2016.

24. Van Beneden K, Geers C, Pauwels M, Mannaerts I, Verbeelen D, van Grunsven LA and Van den Branden C: Valproic acid attenuates proteinuria and kidney injury. J Am Soc Nephrol 22. $1863-1875,2011$

25. Noh H, Oh EY, Seo JY, Yu MR, Kim YO, Ha H and Lee HB: Histone deacetylase-2 is a key regulator of diabetes- and transforming growth factor-beta1-induced renal injury. Am J Physiol Renal Physiol 297: F729-F739, 2009.
26. Sun XY, Qin HJ, Zhang Z, Xu Y, Yang XC, Zhao DM, Li XN and Sun L: Valproate attenuates diabetic nephropathy through inhibition of endoplasmic reticulum stressinduced apoptosis. Mol Med Rep 13: 661-668, 2016.

27. Wade PA: Transcriptional control at regulatory checkpoints by histone deacetylases: Molecular connections between cancer and chromatin. Hum Mol Genet 10: 693-698, 2001.

28. Liu R: The real culprit behind diabetic nephropathy: Impaired renal autoregulation? Physiol Rep 5: e13138, 2017.

29. Gardner BM and Walter P: Unfolded proteins are Ire1-activating ligands that directly induce the unfolded protein response. Science 333: 1891-1894, 2011

30. Ma Y and Hendershot LM: The unfolding tale of the unfolded protein response. Cell 107: 827-830, 2001.

31. Dai H, Liu Q and Liu B: Research progress on mechanism of podocyte depletion in diabetic nephropathy. J Diabetes Res 2017: 2615286, 2017.

32. Cao Y, Hao Y, Li H, Liu Q, Gao F, Liu W and Duan H: Role of endoplasmic reticulum stress in apoptosis of differentiated mouse podocytes induced by high glucose. Int J Mol Med 33: 809-816, 2014.

33. Zhu Y, Cui H, Xia Y and Gan H: RIPK3-mediated necroptosis and apoptosis contributes to renal tubular cell progressive loss and chronic kidney disease progression in rats. PLoS One 11: e0156729, 2016.

34. Luo S, Mao C, Lee B and Lee AS: GRP78/BiP is required for cell proliferation and protecting the inner cell mass from apoptosis during early mouse embryonic development. Mol Cell Biol 26 5688-5697, 2006

35. Schram AW, Baas R, Jansen PW, Riss A, Tora L, Vermeulen M and Timmers HT: A dual role for SAGA-associated factor 29 (SGF29) in ER stress survival by coordination of both histone $\mathrm{H} 3$ acetylation and histone $\mathrm{H} 3$ lysine-4 trimethylation. PLoS One 8: e70035, 2013.

36. Ngwa CJ, Kiesow MJ, Papst O, Orchard LM, Filarsky M, Rosinski AN, Voss TS, Llinás M and Pradel G: Transcriptional profiling defines histone acetylation as a regulator of gene expression during human-to-mosquito transmission of the Malaria parasite plasmodium falciparum. Front Cell Infect Microbiol 7: 320, 2017

37. Zhang $\mathrm{J}$ and Zhong Q: Histone deacetylase inhibitors and cell death. Cell Mol Life Sci 71: 3885-3901, 2014.

38. Zhao H, Alam A, Soo AP, George AJT and Ma D: Ischemiareperfusion injury reduces long term renal graft survival: Mechanism and beyond. EBioMedicine 28: 31-42, 2018.

39. Seet LF, Toh LZ, Finger SN, Chu SW, Stefanovic B and Wong TT: Valproic acid suppresses collagen by selective regulation of Smads in conjunctival fibrosis. J Mol Med (Berl) 94: 321-334, 2016.

40. Feng J, Li S and Chen H: Tanshinone IIA ameliorates apoptosis of cardiomyocytes induced by endoplasmic reticulum stress. Exp Biol Med (Maywood) 241: 2042-2048, 2016.

41. Chen Y, Tang Y, Xiang Y, Xie YQ, Huang XH and Zhang YC: Shengmai injection improved doxorubicin-induced cardiomyopathy by alleviating myocardial endoplasmic reticulum stress and caspase-12 dependent apoptosis. Biomed Res Int 2015: 952671, 2015.

42. Zhang Z, Tong N, Gong Y, Qiu Q, Yin L, Lv X and Wu X: Valproate protects the retina from endoplasmic reticulum stress-induced apoptosis after ischemia-reperfusion injury. Neurosci Lett 504: 88-92, 2011.

43. Spotswood HT and Turner BM: An increasingly complex code. J Clin Invest 110: 577-582, 2002.

44. Baumeister P, Dong D, Fu Y and Lee AS: Transcriptional induction of GRP78/BiP by histone deacetylase inhibitors and resistance to histone deacetylase inhibitor-induced apoptosis. Mol Cancer Ther 8: 1086-1094, 2009.

45. Baumeister P, Luo S, Skarnes WC, Sui G, Seto E, Shi Y and Lee AS: Endoplasmic reticulum stress induction of the Grp78/BiP promoter: Activating mechanisms mediated by YY1 and its interactive chromatin modifiers. Mol Cell Biol 25: 4529-4540, 2005.

46. Bown CD, Wang JF, Chen B and Young LT: Regulation of ER stress proteins by valproate: Therapeutic implications. Bipolar Disord 4: 145-151, 2002.

47. Bush EW and McKinsey TA: Protein acetylation in the cardiorenal axis: The promise of histone deacetylase inhibitors. Circ Res 106: 272-284, 2010.

48. Bannister AJ and Kouzarides T: Regulation of chromatin by histone modifications. Cell Res 21: 381-395, 2011.

49. Choudhary C, Kumar C, Gnad F, Nielsen ML, Rehman M, Walther TC, Olsen JV and Mann M: Lysine acetylation targets protein complexes and co-regulates major cellular functions. Science 325: 834-840, 2009. 